\title{
Editorial
}

\section{Coincidences: A tale of two genes, ami-1 and upr-1}

Of the editors, chief editors or, grander still, editors-in-chief of the Academy's eleven journals, four (Rohini Godbole, Chanda Jog, Gadadhar Misra and I) were contemporaries in the late 70s/early 80s at the State University of New York at Stony Brook (now Stony Brook University). If that is not one crazy coincidence, here is another: I knew the people living at \#1, 234 E5th Street, NYC. In those Manhattan-centric years, this brought exquisite name-dropping rights.

Coincidence \#3 brings in a whiff of the preternatural. For 18 years my laboratory had studied repeatinduced point mutation (RIP), a 'genome defense' process of fungi that occurs during a sexual cross and induces mutations in duplicated DNA sequences. RIP was suppressed in Neurospora crassa crosses heterozygous for chromosome segment duplications $(D p)$, but only if the $D p$ was relatively large $(>270 \mathrm{kbp})$. Later, in a tangential extension of the study, we defined the breakpoints of the $D p$-generating chromosome rearrangements onto the genome sequence, and found one rearrangement that disrupted the Neurospora homologue of the Podospora anserina gene ami-1 (see J. Biosci. 35 539-546; December 2010). Only a few years previously had a group in France reported that in Podospora, ami-1-homozygous mutant crosses display an increase in RIP efficiency. That we had stumbled en passant upon a Neurospora mutation in the only other gene implicated in RIP was taken as a sign of encouragement from the 'Great Bread Mould (GBM)'.

Maybe we should have propitiated the GBM, because my coincidence \#4 came in the form of a scientist's worst nightmare - an experiment that suddenly and inexplicably stops being reproducible. A PhD student had mapped a suppressor of RIP to the Neurospora upr-1 locus. (What compounds matters is the fact that the ami-1-homologue and upr-1 are adjacent loci, only $5 \mathrm{kbp}$ apart, but as far as we know, their cheek-by-jowl propinquity is just another coincidence.) He then obtained disruptions of the upr-1 gene via targeted integration of transforming DNA, and showed that loss of the upr-1 allele was correlated with loss of RIP suppression. We published these findings in a paper dedicated to the memory of my hero, David D Perkins. It was the best of times.

A few months later, two other students wanted to learn how to target gene disruptions in Neurospora. I suggested that they repeat the upr-1 disruption experiment from scratch. It was $\mathrm{Al}$ Hershey's idea of heaven - to have one experiment that works, and keep doing it all the time (see Judson HF, The eighth day of creation: The makers of the revolution in biology). But now the results were hellish: loss of upr-1 did not result in loss of suppression. Apparently, now the upr-1 allele was not the suppressor. Grimly, we retracted the earlier result and withdrew a manuscript that was in review, as it turns out, in this journal. I recalled George Bernard Shaw, what is life but a series of inspired follies? Was the earlier result simply a malign coincidence, or is there an explanation for the same experiment giving different results when repeated? It was the worst of times.

Time heals, and at least occasionally, doing the right thing has its rewards. Singed by our 'bonfire of the vanities', we re-checked hundreds of old crosses (progeny ascospores from Neurospora crosses can be stored for several years), and in so doing we made a startling discovery - ascospores from crosses compromised in genome defense have reduced long-term viability (accepted in Fungal Genetics and Biology 2011). To rework an old aphorism, the road to discovery is rutted with mistakes and happenstance.

DuRGadAs P KaSBEKAR Editor 